\title{
Effect of evidence-based nursing on the application of ultrasonic emulsification operation for cataract and the postoperative rehabilitation indicators
}

\author{
Yanqin TU ${ }^{1}$, Jianmin $\mathrm{DENG}^{1}$, Liang FANG ${ }^{1}$, Yongmei ZHANG ${ }^{1}$, Hongmei YING ${ }^{1}$, Qiuzhen SUN ${ }^{1 *}$ (iD
}

\begin{abstract}
To explore the effect of evidence-based nursing on the application of ultrasonic emulsification operation for cataract and the postoperative rehabilitation indicators. 110 cataract patients were included. All of them received ultrasonic emulsification operation for cataract and were divided into the control group $(n=55)$ and the observation group $(n=55)$ by random number method. The control group received routine nursing. The visual acuity, intraocular pressure, quality of life and adverse reactions of patients in both groups after discharge were compared and evaluated. The clinical effective rate( $92.73 \%)$, postoperative visual acuity $(0.68 \pm 0.05)$ and SF-36 score $(82.37 \pm 4.13)$ in the observation group were higher than those in the control group $(76.36 \%)$, $(0.55 \pm 0.02)$ and $(74.06 \pm 3.42)$, Conclusion: Evidence-based nursing can effectively improve the curative effect and postoperative vision, and improve the quality of life. The intraocular pressure in the observation group $(9.95 \pm 2.46) \mathrm{mmHg}$ and the incidence of adverse reactions $(29.09 \%)$ were lower than those in the control group $(11.03 \pm 3.11) \mathrm{mmHg}$ and $10.91 \%(\mathrm{P}<0.05)$. It is of distinct effect to carry out evidence-based nursing for the cataract patients receiving emulsification therapy. It effectively improves the effect of surgical treatment, reduce the occurrence of adverse reactions, so as to improve the quality of life and promote rapid physical recovery, maintaining clinical application value.
\end{abstract}

Keywords: cataract; ultrasonic emulsification; evidence-based nursing; adverse reactions.

Practical Application: Some studies have confirmed that cataract can make cloudy lens interfere with light, resulting in patients with retina can not accurately image, monocular diplopia, glare, blurred vision and other clinical symptoms.

\section{Introduction}

Cataract, as a common eye disease in clinic, refers to the deterioration of the patient's own body function and abnormal metabolic system, leading to persistent aging of the lens, and then affect the quality of life and physical and mental health of patients. At present, cataract is mainly treated by surgical methods, including ultrasonic emulsification operation and non-ultrasonic emulsification operation. Non-ultrasonic emulsification cataract extraction (NPHCE) is a common ophthalmic operation in the past. It has the advantages of low cost and convenient operation. It can obtain better incision closure, but there are many complications. Since the ultrasonic emulsification apparatus was firstly invented by Kelman in 1967, the current treatment of cataract has changed from traditional cataract surgery to emulsification surgery, and the ideal therapeutic effect is achieved mainly through implantation of intraocular lens (Li et al., 2018; Kirkwood et al., 2006). However, the operation is invasive and patients need a long time for postoperative recovery. Therefore, the nursing at this stage is particularly critical for prognosis. Evidence-based nursing refers to the systematic use of nursing experience of domestic and external cataract patients receiving ultrasonic emulsification operation through evidence-based evidence, and the combination of the obtained evidence with the needs of patients, so as to reduce the risks for patients. Studies have pointed out that the effect of evidence-based nursing after surgery for elderly cataract patients is better than routine nursing, and appropriate nursing methods can effectively improve the quality of nursing and the satisfaction of patients (Fu \& Zhao, 2011). However, there are few reports about the combined evidence-based nursing after operation. In order to improve the prognosis of patients after cataract surgery and to find the best quality nursing methods, a total of 110 cataract patients admitted to the ophthalmology department of our hospital from January 2020 to December 2020 were studied in this paper. These patients were randomly divided into two groups for routine nursing and evidence-based nursing, with detailed reports as following.

\section{Materials and methods}

\subsection{General data}

A total of 110 cataract patients admitted to the ophthalmology department of our hospital from January 2020 to December 2020 were included. All the patients received ultrasonic emulsification operation for cataract and were divided into the control group $(n=55)$ and the observation group $(n=55)$ by random number method. The comparison of the general data of both groups shows the differences were comparable $(P>0.05)$, as shown in Table 1 . This study was in compliance with relevant medical 
Table 1. Comparison of the General Data of both Groups.

\begin{tabular}{|c|c|c|c|c|c|c|c|}
\hline \multirow{2}{*}{ Group } & \multirow{2}{*}{$\mathrm{n}$} & \multicolumn{2}{|c|}{ Gender [n (\%)] } & \multicolumn{2}{|c|}{ Side of Sick Eye [n (\%)] } & \multirow{2}{*}{ Mean Age (Years) } & \multirow{2}{*}{$\begin{array}{c}\text { Mean Course of } \\
\text { Disease (Years) }\end{array}$} \\
\hline & & Male & Female & Single Side & Both Sides & & \\
\hline Observation group & 55 & $35(63.64)$ & $20(36.36)$ & $45(81.82)$ & $10(18.18)$ & $64.27 \pm 2.75$ & $2.32 \pm 0.64$ \\
\hline Control group & 55 & $36(65.45)$ & $19(34.55)$ & $46(83.64)$ & $9(16.36)$ & $65.23 \pm 2.55$ & $2.30 \pm 0.58$ \\
\hline$x^{2} / t$ & - & \multicolumn{2}{|c|}{0.072} & \multicolumn{2}{|c|}{0.116} & 1.898 & 0.172 \\
\hline$P$ & - & \multicolumn{2}{|c|}{0.789} & \multicolumn{2}{|c|}{0.734} & 0.060 & 0.864 \\
\hline
\end{tabular}

Note: $\mathrm{x} 2$ is chi-square test, $\mathrm{t}$ is student $\mathrm{t}$ test, $\mathrm{n}$ is the number of cases, and $\mathrm{p}$ is the probability that the original hypothesis is true.

ethical standards, and all patients voluntarily participated in the study and signed the Informed Consent Form.

\subsection{Inclusion and exclusion criteria}

Inclusion criteria: (1) clinically relevant examinations meet the diagnostic criteria for eye diseases (Kamiya et al., 2018); (2) preoperative visual acuity below 0.03 ; (3) no contraindications to surgery. Exclusion criteria: (1) patients with mental illness or cognitive impairment; (2) patients with severe insufficiency of heart, liver, kidney, lung and other organs and malignant tumors; (3) lack of clinical data and withdrawal.

\subsection{Methods}

Ultrasonic emulsification operation was performed in both groups. The whole cornea was removed and the anterior lens capsule was removed. After cataract extraction, cortex was pulverized by ultrasound and intraocular lens was implanted.

The control group received routine nursing plan: provide the patients with nursing services in strict compliance with the ophthalmic nursing procedures and regulations, such as medication guidance, eye nursing and health education et al. The patients with abnormal conditions are handled timely, and the postoperative precautions are instructed, the family members are informed to pay more attention, and the patients are provided with a quiet rest environment. To deal with abnormal situation in time, strictly carry out aseptic operation during operation, improve the safety of operation.

The observation group received evidence-based nursing plan: preoperative nursing: 1) Eyelid Cleaning. before surgery, eye drops of broad-spectrum antibiotic were used 4-6 times/d, and $5 \%$ povidone-iodine was used to flush the conjunctival sac. The patients were asked not to cough, sneeze, etc. Patients should use tongue to withstand the roof of mouth to relieve impulses, so as to ensure the smooth operation. Carefully affix eyelashes and eyelids to ensure minimal exposure to the surgical field of view; 2) Health Education. conduct preoperative disease education for patients, actively indicate surgical characteristics to patients, and carefully introduce disease etiology, surgical indications, surgical precautions and equipment to patients and their families in written form and video popular science form, so as to effectively improve the patients' cognition level of the disease; 3) Preoperative Examination. The general examinations, including blood glucose and blood pressure, were performed before operation. If the patient has a history of perennial hypertension, the blood pressure should be controlled below $19 / 12 \mathrm{Kpa}$. All the results were carefully checked to see if they were perfect one day before surgery. Patients with tension induced hypertension were treated with counseling, and the operation was performed after the blood pressure turned normal. Nursing during operation: the aseptic operation was strictly implemented during the operation to improve the safety. Nursing after operation: 1) Anti-infective treatment. Follow the doctor's advice for anti-infection treatment and inject cefuroxime sodium into the eyes to prevent infection; 2) Complication care. tell patients to take a full rest after the operation, do not excessive activity, rubbing the eyes, sneezing, coughing and suddenly bow, etc. For intraocular bleeding during the operation, the head should be properly raised or taken semi-decubitus position to avoid the impact on postoperative vision. The patients could get out of bed 24 hours after the operation, and should ensure that the surgical dressing was dry and clean when eating and washing. When using eye drops, the patients were instructed to use the correct method. The patients were told to make sure the hands were clean before applying eye drops, and do not press the eyeball vigorously after applying eye drops, so as to avoid wound bleeding or displacement of intraocular lens; 3) strengthen psychological nursing. eliminate patients' fear, anxiety and anxiety, and comfort patients; 4) nursing of complications: pay close attention to the postoperative symptoms of the patients, most of the patients suffered complications due to the residual viscoses, ocular inflammation, such as high intraocular pressure, corneal edema, etc., when the doctor should be informed in time for treatment; 5) dietary requirements: develop a scientific and reasonable diet plan to encourage patients to eat more fresh fruits and melons rich in vitamins. The elderly and infirm could be granted semi-liquid food firstly. The combination of hypertension could take the food with low fat and low salt; 6) discharge guidance: the nursing team would make the discharge instruction manual with easy to understand words and pictures, which mainly included the application method of eye drops, return visit time, precautions for activities and rest, eye hygiene, nutrition guidance, attention to warmth, emotional control, etc. The study was approved by our hospital. Informed consent was obtained.

\subsection{Observation index}

(1) Efficacy evaluation: Combined with the vision questionnaire (Jalali, 2018), the postoperative efficacy of patients was evaluated. The higher the score, the worse the efficacy. Excellent: the patients' visual acuity level returned to normal, and the clinical symptoms such as light phobia, blurred vision and dark object color were completely eliminated, and the questionnaire score was lower than 15 points; valid: the visual acuity of patients tended to be 
normal, clinical symptoms improved, and questionnaire scores ranged from 15 to 25 points; invalid: those failing to meet the above description. The patients' clinical efficacy $=$ excellent rate + effective rate;

(2) The visual acuity and intraocular pressure were recorded before discharge for patients in the two groups, and the quality of life was assessed according to the evaluation standards for quality of life (SF-36) (Bilbao et al., 2009), which mainly included mental health, social function, physical health and emotional function, etc. The higher the score, the better the recovery;

(3) The adverse reactions during postoperative nursing in the two groups were counted.

\subsection{Statistical analysis}

The statistical software SPSS21.0 was used for analysis. The counting data were expressed as " $n$ " and "(\%)", the measurement data as $(x \pm s), \chi^{2}$ and " $t$ " tests were performed respectively. $P<0.05$ showed a statistically significant difference.

\section{Results}

\subsection{Comparison of clinical effective rate between two groups of patients}

The clinical effective rate (92.73\%) in the observation group was higher than that in the control group (76.36\%), and the difference was statistically significant $(P<0.05)$, it shows that evidence-based nursing can effectively alleviate symptoms and improve efficacy. As shown in Table 2.

\subsection{Comparison of visual acuity, intraocular pressure and life quality between the two groups of patients before discharge}

After treatment, the visual acuity and scores of quality of life in control group were lower than those in observation group $(P<0.05)$, while the intraocular pressure in control group was higher than that in observation group $(P<0.05)$. The results indicate that evidence-based nursing can improve vision, intraocular pressure and quality of life, and improve the postoperative recovery. As shown in Table 3.

\subsection{Comparison of postoperative adverse reactions between the two groups of patients}

There were 16 cases $(29.09 \%)$ with adverse reactions in the control group and 6 cases with adverse reactions in the observation group (10.91\%), and the difference between both groups was statistically significant $(P<0.05)$. It indicated that the adverse reactions caused by evidence-based nursing were less than those of conventional nursing, which could improve the safety of nursing. As shown in Table 4.

\section{Discussion}

Some studies have confirmed that cataract can make cloudy lens interfere with light, resulting in patients with retina can not accurately image, monocular diplopia, glare, blurred vision and other clinical symptoms (Kumar et al., 2018; Foster et al., 2018). At present, the pathogenesis of cataract is not clear, but a large number of studies have shown that it may be closely related to heredity, lens metabolism, hypertension, diabetes and other factors (Ramke et al., 2017). Patients have a long recovery time after surgery, so quality care is essential. High-quality nursing care can actively reduce postoperative complications and help patients recover early. In Barnett \& Neves (2018) and Ying et al. (2018), it was found that the visual acuity of non- ultrasonic emulsification operation was much lower than that of ultrasonic emulsification operation, mainly due to the damage of atrial horn and blood chamber barrier. Meanwhile, Gong et al. (2018) report showed that the incidence of iris collapse and corneal edema in non-ultrasonic emulsification was higher than that in ultrasonic emulsification, reaching $23.45 \%$.

Therefore, ultrasonic emulsification operation is more suitable for cataract surgery. However, because the eyes are more fragile and the age of the patients is generally high, so no matter what

Table 2. Comparison of Clinical Effective Rate between Two Groups of Patients [n, (\%)].

\begin{tabular}{cccccc}
\hline Group & $\mathrm{n}$ & Excellent & Valid & Invalid & Total Effective Rate \\
\hline Control group & 55 & $24(43.64)$ & $18(32.73)$ & $13(23.64)$ & $42(76.36)$ \\
Observation group & 55 & $28(50.91)$ & $23(41.82)$ & $4(7.27)$ & $51(92.73)$ \\
$x^{2}$ & & & & 10.254 \\
$P$ & & & & 0.001 \\
\hline
\end{tabular}

Note: $\mathrm{x} 2$ is chi-square test, $\mathrm{n}$ is the number of cases, and $\mathrm{p}$ is the probability that the original hypothesis is true.

Table 3. Comparison of Visual Acuity, Intraocular Pressure and Life Quality between the Two Groups of Patients before Discharge ( ${ }^{-} \mathrm{x} \pm \mathrm{s}$ ).

\begin{tabular}{ccccc}
\hline Group & Cases & Postoperative Visual Acuity & Postoperative Intraocular Pressure $(\mathrm{mmHg})$ & SF-36 Score (points) \\
\hline Observation group & 55 & $0.68 \pm 0.05$ & $9.95 \pm 2.46$ & $82.37 \pm 4.13$ \\
Control group & 55 & $0.55 \pm 0.02$ & $11.03 \pm 3.11$ & $74.06 \pm 3.42$ \\
$t$ & & 17.903 & 2.020 & 11.493 \\
$P$ & & 0.000 & 0.046 & 0.000 \\
\hline
\end{tabular}

Note 1: The visual acuity of the observation group was higher than that of the control group, and the intraocular pressure was lower than the control groups. The SF-36 score was higher in observation group than in control group. It showed that evidence-based nursing improved visual acuities, intraocular pressure, and the quality of life. Note2: $t$ is student $t$ test, $n$ is the number of cases, and $\mathrm{p}$ is the probability that the original hypothesis is true. 
Table 4. Comparison of Postoperative Adverse Reactions between the Two Groups of Patients [n,(\%)].

\begin{tabular}{|c|c|c|c|c|c|c|c|}
\hline Group & $\mathrm{n}$ & $\begin{array}{c}\text { Corneal } \\
\text { Edema }\end{array}$ & $\begin{array}{c}\text { Intraocular } \\
\text { Hypertension }\end{array}$ & Endoophthalmitis & Iris Prolapse & Hyphema & $\begin{array}{c}\text { Total Incidence } \\
\text { Rate }\end{array}$ \\
\hline Control group & 55 & $6(10.91)$ & $4(7.27)$ & $2(3.64)$ & $1(1.82)$ & $3(5.45)$ & $16(29.09)$ \\
\hline Observation group & 55 & $3(5.45)$ & $1(1.82)$ & $1(1.82)$ & 0 & $1(1.82)$ & $6(10.91)$ \\
\hline$x^{2}$ & & & & & & & 10.329 \\
\hline$P$ & & & & & & & 0.001 \\
\hline
\end{tabular}

Note: The symptoms of corneal edema, Intraocular Hypertension, Endophthalmitis, Iris Prolapse and Hyphema in the observation group were lower than those in the control group, indicating that evidence-based nursing can help patients reduce adverse reactions. Note: $\mathrm{x}^{2}$ is chi-square test, $\mathrm{n}$ is the number of cases, and $\mathrm{p}$ is the probability that the original hypothesis is true.

kind of surgical treatment will produce certain damage to the body. Proper nursing can effectively improve the prognosis of patients and improve the ability of rehabilitation.

The visual acuity of cataract patients after operation is generally weak, and due to their own characteristics, cognitive function decline, self-protection ability decline and so on. Evidence-based nursing can provide effective protection according to the psychological characteristics and cognitive ability of patients, promote more professional and safe nursing process. The results of this study showed that the efficacy and postoperative IOP, visual acuity and quality of life in the observation group were higher than those in the control group, consistent with Morrell's (2001) study. It indicates that the scheme is feasible and effective. Zhang et al. (2018) noted that patients with appropriate evidence-based care, Can effectively improve the quality of life of patients, but also improve the ability of self-control, so as to avoid vascular contraction disorder, control vascular regulation center in a stable state, and then effectively control intraocular pressure, protect vision. After IOL implantation in the operating room, antibiotic eye drops, saline should be used to gently clean the eyelid, and eye pads should be covered. In the observation group, the nursing process strictly followed the principle of asepsis, which effectively guaranteed the safety of patients. At the same time, IOP and visual acuity were measured regularly in order to reflect the rehabilitation effect of the patients.

In addition, this study selected evidence-based nursing from the previous nursing results, not only summarized the preoperative and postoperative care of patients, And prone to complications of display and nursing, including corneal edema, high intraocular pressure, endophthalmitis, iris prolapse, Hyphema. By strengthening nursing staff for complications of symptomatic nursing measures, can effectively improve nursing staff quality, but also can effectively guide patients to master the correct risk prevention skills. At the same time, this study introduced psychological nursing, dietary nursing and post-discharge guidance on the basis of the previous studies, aiming at improving patients' confidence in recovery, alleviating patients' depression and anxiety, and ensuring postoperative recovery and improving quality of life (Zhao et al., 2018).

The ultrasonic emulsification operation and non-ultrasonic emulsification operation are the main therapies for clinical cataract patients at present. The non-ultrasonic emulsification operation cheap and easy to operate, which is suitable for primary hospitals or interns. Some studies have shown that the visual acuity recovery of patients after non-ultrasonic emulsification operation is lower than that after ultrasonic emulsification operation, for the former may damage ocular atrial horn tissue and blood atrial barrier to a certain extent (Ying et al., 2018). Meanwhile, studies have shown that the incidence of ultrasonic emulsification operation in the treatment of iris collapse and corneal edema is higher than that of non-ultrasonic emulsification operation, reaching 23.45\% (Gong et al., 2018). However, due to the fragile eyes and the relatively high age of surgical patients, whatever surgical treatment is used, it will cause certain damage to the body. Therefore, appropriate nursing for patients can effectively improve the prognosis of patients and improve their rehabilitation ability.

The nursing plan should be made according to the patients' own physical state and tolerance degree, the difference of operation time, et al. The study introduced evidence-based nursing for analysis, and the results showed that the observation group with evidence-based nursing was higher than the control group in terms of curative effect, postoperative intraocular pressure, vision, quality of life and other recovery, which was consistent with the research results of Morrell (2001). And suggested that the plan is highly feasible and effective. The patients with appropriate evidence-based nursing can effectively improve the quality of life, improve the self-control ability, so as to avoid the occurrence of vascular contraction disorder, control the vascular regulation center in a stable state, and effectively control the intraocular pressure and protect vision. After implantation of intraocular lens in the operating room, patients need to use antibiotic eye drops, normal saline to gently clean the eyelid, and also cover the eye pad. The aseptic principle was strictly implemented for the patients in the observation group during the operation to improve the safety (Zhang et al., 2018). Meanwhile, the intraocular pressure and visual acuity of the patients were regularly measured in this paper, aiming to further reflect the rehabilitation effect of the patients. Patients with cataract generally show poor vision after surgery, and suffer from cognitive function decline and self-protection ability decline due to their own characteristics. Evidence-based nursing can provide effective guarantee according to patients' psychological characteristics and cognitive ability, and promote more professional and safe nursing. In addition, the evidence-based nursing in the observation group could be used effectively according to the possible intraoperative complications of patients, including corneal edema, high intraocular pressure, endophthalmitis, iris prolapses, hphema et al. By strengthening the nursing staff's symptomatic nursing measures for complications, the nursing quality can be effectively improved, and patients can also be effectively guided to master the correct risk prevention 
skills (Zhao et al., 2018). Meanwhile, the evidence-based nursing for patients' psychological state of symptomatic nursing, can effectively improve patients' confidence in rehabilitation, relieve the state of depression and anxiety, ensure the postoperative rehabilitation effect, and improve the quality of life. There were 16 cases $(29.09 \%)$ of adverse reactions in the control group. There were 6 cases $(10.91 \%)$ in the observation group $(\mathrm{P}<0.05)$. It is suggested that strengthening the guidance of life and diet can help the patients to control the postoperative complications and improve the visual acuity and intraocular pressure. In diet according to the patient's physical condition to provide a scientific and reasonable plan, release and explain after discharge need to pay attention to aspects, can improve patients SF-36 score, and then accelerate physical recovery. However, at present, the level of health education for senile cataract patients is still unequal, and the overall quality of doctors is uneven. According to the concept of evidence-based nursing, this study will carry on multi-directional management to this kind of patients, in order to study the suitable nursing model for China.

\section{Conclusion}

In conclusion, the cataract patients are accompanied with decreased vision and poor physical quality, which is prone to complications after surgery and affect the quality of life. The evidence-based nursing could improve the effect of surgical treatment, reduce the occurrence of adverse conditions, so as to improve the quality of life remarkably, being worthy of clinical application and promotion.

\section{Acknowledgements}

This study was funded by the Jiangxi Provincial Health Commission Project: (202130518), Application of "one disease, one product" program in nursing of patients with chronic sinusitis undergoing endoscopic surgery.

\section{References}

Barnett, S. R., \& Neves, S. E. (2018). Cataract surgery in the elderly. In S. R. Barnett \& S. E. Neves (Eds.), Perioperative care of the elderly patient. Cambridge: Cambridge University Press. http://dx.doi. org/10.1017/9781316440568.

Bilbao, A., Quintana, J. M., Escobar, A., García, S., Andradas, E., Baré, M., \& Elizalde, B. (2009). Responsiveness and clinically important differences for the VF-14 index, SF-36, and visual acuity in patients undergoing cataract surgery. Ophthalmology, 116(3), 418-424.e1. http://dx.doi.org/10.1016/j.ophtha.2008.11.020. PMid:19168220.

Foster, G. J. L., Allen, Q. B., Ayres, B. D., Devgan, U., Hoffman, R. S., Khandelwal, S. S., Snyder, M. E., Vasavada, A. R., \& Yeoh, R. (2018). Phacoemulsification of the rock-hard dense nuclear cataract: options and recommendations. Journal of Cataract and Refractive
Surgery, 44(7), 905-916. http://dx.doi.org/10.1016/j.jcrs.2018.03.038. PMid:29960655.

Fu, X. X., \& Zhao, C. L. (2011). Nursing care of cataract phacoemulsification in elderly patients over 80 years old. Journal of Clinical Ophthalmology, $19,82-83$.

Gong, D. H., Liu, J. F., Zhao, X., \& Zhang, L. (2018). The effect of nursing intervention on preoperative cataract. Medicine, 97(42), e12749. http://dx.doi.org/10.1097/MD.0000000000012749. PMid:30334960.

Jalali, S. (2018). Post-cataract surgery endophthalmitis. In T. Das (Ed.), Endophthalmitis: a guide to diagnosis and management (Chap. 7, pp. 81-92). Singapore: Springer. http://dx.doi.org/10.1007/978981-10-5260-6_7.

Kamiya, K., Iijima, K., Nobuyuki, S., Mori, Y., Miyata, K., Yamaguchi, T., Shimazaki, J., Watanabe, S., \& Maeda, N. (2018). Predictability of intraocular lens power calculation for cataract with keratoconus: a multicenter study. Scientific Reports, 8(1), 1312. http://dx.doi. org/10.1038/s41598-018-20040-w. PMid:29358627.

Kirkwood, B. J., Pesudovs, K., Latimer, P., \& Coster, D. J. (2006). The efficacy of a nurse-led preoperative cataract assessment and postoperative care clinic. The Medical Journal of Australia, 184(6), 278-281. http://dx.doi.org/10.5694/j.1326-5377.2006.tb00237.x. PMid:16548832.

Kumar, S. G. P., Mondal, A., Vishwakarma, P., Kundu, S., Lalrindiki, R., \& Kurian, E. (2018). Factors limiting the Northeast Indian elderly population from seeking cataract surgical treatment: evidence from Kolasib district, Mizoram, India. Indian Journal of Ophthalmology, 66(7), 969-974. http://dx.doi.org/10.4103/ijo. IJO_1184_17. PMid:29941741.

Li, E., Margo, C. E., \& Greenberg, P. B. (2018). Cataract surgery outcomes in the very elderly. Journal of Cataract and Refractive Surgery, 44(9), 1144-1149. http://dx.doi.org/10.1016/j.jcrs.2018.05.025. PMid:30055955.

Morrell, G. (2001). Effect of structured preoperative teaching on anxiety levels of patients scheduled for cataract surgery. Insight, 26(1), 4-9. http://dx.doi.org/10.1067/min.2001.113201. PMid:11246179.

Ramke, J., Gilbert, C. E., Lee, A. C., Ackland, P., Limburg, H., \& Foster, A. (2017). Effective cataract surgical coverage: an indicator for measuring quality-of-care in the context of Universal Health Coverage. PLoS One, 12(3), e0172342. http://dx.doi.org/10.1371/ journal.pone.0172342. PMid:28249047.

Ying, X., Gong, D. H., Zhao, X., Wei, Q., Zhang, Y., Wang, J., \& Hao, L. (2018). Effect of comprehensive nursing intervention in perioperative period of glaucoma combined with cataract phacoemulsification and intraocular lens implantation. China Modern Doctor, 56, 154-156.

Zhang, D., Fan, Z., Gao, X., Huang, W., Yang, Q., Li, Z., Lin, M., Xiao, H., \& Ge, J. (2018). Illness uncertainty, anxiety and depression in Chinese patients with glaucoma or cataract. Scientific Reports, 8(1), 11671. http://dx.doi.org/10.1038/s41598-018-29489-1. PMid:30076311.

Zhao, Y., Xu, L. F., Zhao, Y. N., \& Hospital, B. J. (2018). Effect of the NBASS-APS on the anxiety or depression and nursing satisfaction caused by the postoperative pain in the cataract patient. Hebei Medicine, 24, 339-343. 